\title{
Establishing a Disruptive New Capability for NASA to Fly UAV's into Hazardous Conditions
}

\author{
Jay Ely*a, Truong Nguyen ${ }^{\mathrm{a}}$, Jennifer Wilson ${ }^{\mathrm{b}}$, Robert Brown ${ }^{\mathrm{b}}$, Sean Laughter ${ }^{\mathrm{a}}$, Ed Teets ${ }^{\mathrm{c}}$, Allen \\ Parkerc, "Patrick" Hon Man Chan", Lance Richards ${ }^{c}$

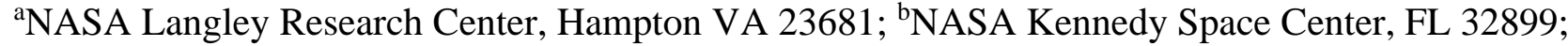 \\ 'NASA Armstrong Flight Research Center, Edwards, CA 93523
}

\begin{abstract}
A 2015 NASA Aeronautics Mission "Seedling" Proposal is described for a Severe-Environment UAV (SE-UAV) that can perform in-situ measurements in hazardous atmospheric conditions like lightning, volcanic ash and radiation. Specifically, this paper describes the design of a proof-of-concept vehicle and measurement system that can survive lightning attachment during flight operations into thunderstorms. Elements from three NASA centers draw together for the SE-UAV concept. 1) The NASA KSC Genesis UAV was developed in collaboration with the DARPA Nimbus program to measure electric field and X-rays present within thunderstorms. 2) A novel NASA LaRC fiber-optic sensor uses Faraday-effect polarization rotation to measure total lightning electric current on an air vehicle fuselage. 3) NASA AFRC's state-of-the-art Fiber Optics and Systems Integration Laboratory is envisioned to transition the Faraday system to a compact, light-weight, all-fiber design.

The SE-UAV will provide in-flight lightning electric-current return stroke and recoil leader data, and serve as a platform for development of emerging sensors and new missions into hazardous environments. NASA's Aeronautics and Science Missions are interested in a capability to perform in-situ volcanic plume measurements and long-endurance UAV operations in various weather conditions. (Figure 1 shows an artist concept of a SE-UAV flying near a volcano.) This paper concludes with an overview of the NASA Aeronautics Strategic Vision, Programs, and how a SE-UAV is envisioned to impact them. The SE-UAV concept leverages high-value legacy research products into a new capability for NASA to fly a pathfinder UAV into hazardous conditions, and is presented in the SPIE DSS venue to explore teaming, collaboration and advocacy opportunities outside NASA.
\end{abstract}

Keywords: UAV, Lightning, Volcanic Ash, Faraday, Fiber-Optic, Weather, Environment



Figure 1. SE-UAV flying near a volcano. (NASA graphic artist concept)

*jay.j.ely@nasa.gov;phone 1757864 1868; fax 1757 864-4234 


\section{INTRODUCTION}

Airplane crews and passengers depend on the experience, senses and creativity of a human pilot to prioritize risk and take appropriate action when unforeseen circumstances occur. To become trusted in the national air space (NAS), UAVs need to compensate for the absence of a pilot with autonomous systems having increased resiliency to upset conditions and improved situational awareness of the environment.

Even with the best weather forecasting tools and planning available, unforeseen circumstances sometimes give way to aviation operations in convective environments, and our understanding of lightning effects on aircraft remains far from complete. Passenger airplanes and UAVs are increasingly-constructed from non-conductive composite materials. Inflight lightning experiments were performed close to 30 years ago, on aluminum aircraft, and were limited to above $5 \mathrm{kft}$ altitude and highly-restricted geographical locations [1]. Actual aircraft lightning strike measurement data are extremely limited due to unsuitable sensors in past flight measurements. Existing test standards are based on ground measurements, but decades-long accumulation of data show the lightning environment to vary with vehicle size, velocity, flight latitude and altitude [2]. Aircraft manufacturers, SAE Committees, Scientists, and the DOD want in-situ lightning data for aerospace vehicle design \& certification, facility protection, flight operations and climate science. Lightning is also a factor in other severe environments (convection/icing/volcanoes) [3]. New severe-environment data are needed for UAV design \& certification, and capabilities for in-situ damage and effects sensing for lightning strikes and kinetic air hazards are needed to establish trust in autonomous flight systems. Figure 2 shows an artist concept of a Genesis UAV flying near the coast of Kennedy Space Center, intercepting a flash of lightning.

The research effort described in this paper will establish a path to enable new missions in the NAS by developing a pathfinder UAV that can perform in-situ meteorology in severe meteorological conditions.

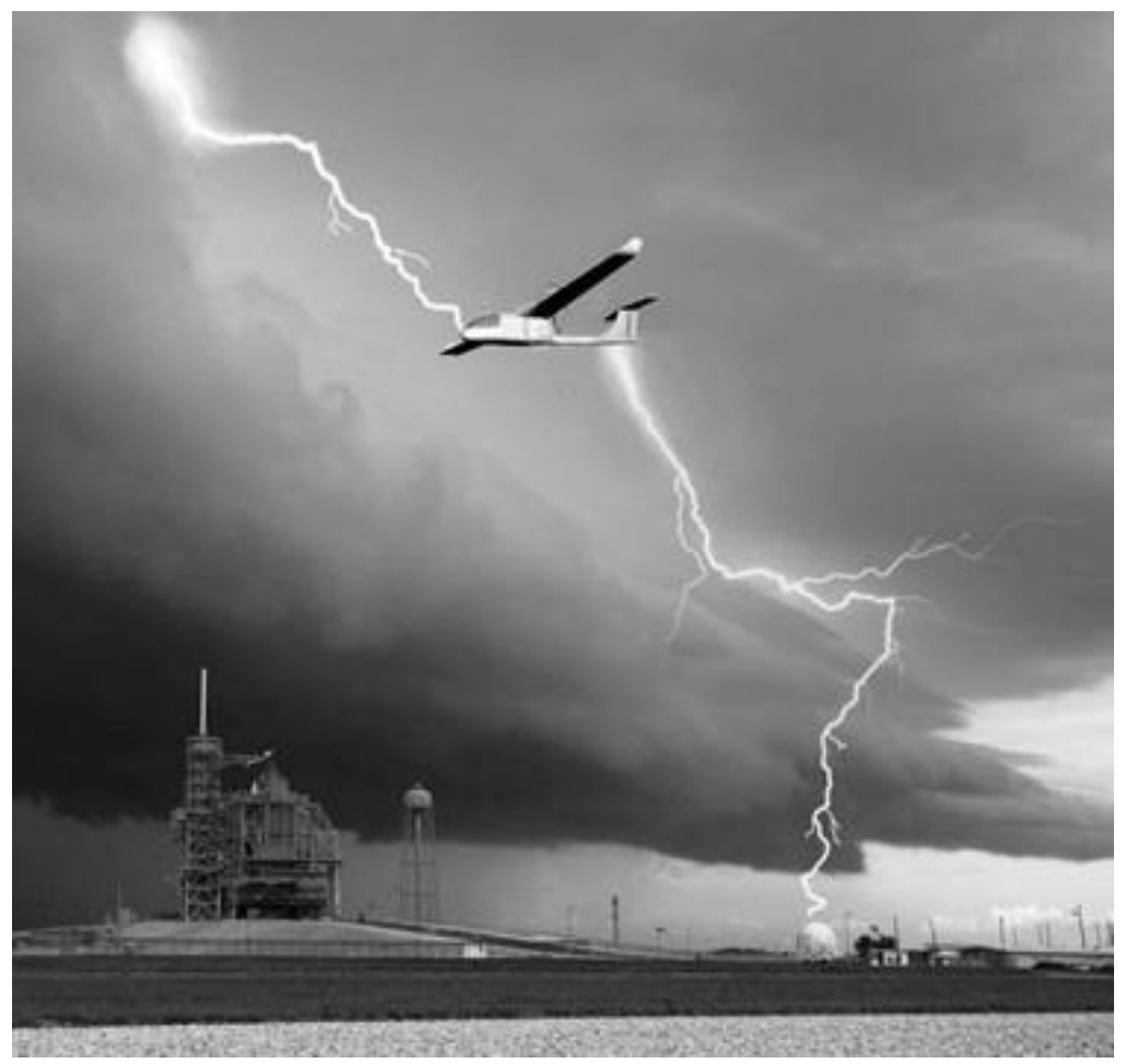

Figure 2. Genesis UAV flying near the coast of Kennedy Space Center, intercepting a flash of lightning. (NASA graphic artist concept) 


\section{TECHNICAL APPROACH}

The NASA KSC Genesis UAV was developed in collaboration with the DARPA Nimbus program to measure electric field and X-rays present within thunderstorms. Genesis will be hardened to withstand direct lightning strikes (i.e. conductive fuselage, fuel system protection, avionics shielding) to a comparable extent with general aviation standard practice, and improved by new measurement data and iterative design \& test. Figure 3 shows the baseline (unshielded) Genesis UAS is constructed from fiberglass and carbon-fiber composite [4].



Figure 3. The baseline (unshielded) Genesis UAS is constructed from fiberglass and carbon-fiber composite, has a 6-ft wing span, is already operational, and was designed and built by NASA KSC Rocket University.

Several prototype Faraday fiber-optic current sensors for lightning measurement applications have been developed at the NASA LaRC E3HIRF (Electromagnetic Environmental Effects and High Intensity Radiated Fields Laboratory). The systems use optical fiber for the sensing element and free-space optics for the interface box, laboratory-grade instrumentation, and can measure lightning electric current up to 400kA. They have been demonstrated in a lightning laboratory and with rocket-triggered lightning at the International Lightning Research and Testing (ICLRT) facility, in Florida, U.S.A. A prototype Faraday fiber optic current sensor and data acquisition system was also installed on the NASA Falcon HU-25C. The HU-25C Faraday sensor installation was tested during three flights near thunderstorms, but the aircraft did not get struck by lightning. No false-triggers were observed, and the installation was proven flightworthy, but project funding was discontinued. Figure 4 shows the HU-25C Faraday system installation. [5]


Figure 4. Optical Fiber installed on HU-25C aircraft, as part of prototype lightning current sensing system.

NASA LaRC lightning sensor researchers will team with NASA AFRC Fiber-Optic instrumentation specialists to reduce the Mass, Volume and Power (MVP) requirements of the Faraday lightning current sensor for use on a small UAV. NASA AFRC's state-of-the-art Fiber Optics and Systems Integration Laboratories are ideally suited to transition the system to an all-fiber design. Researchers from the NASA LaRC Aeronautics Systems Engineering Branch will team 
with the AFRC Fiber Optic and Systems Integration Laboratory to design and build a high-performance SE-UAV instrumentation system using stackable FPGA Mezzanine Card form-factor (sFMC) architecture, greatly reducing MVP of the existing laboratory-grade unit.

Work Plan tasks, as envisioned for a 2015 NASA Aeronautics Mission Directorate (ARMD) "Seedling" proposal are described next. These tasks are expected to be modified as the team, schedule, budget, technology and requirements evolve. A vision of how this effort fits into NASA's Aeronautics mission (and beyond) is provided in Section 3.

\section{Work Plan Task 1: Shielded Genesis Test Vehicle: "Risk Mitigation" Builds}

Expanded copper mesh covering will be applied to a Genesis UAV fuselage, similar to a general-aviation aircraft practice. The aircraft will be flown through electrified clouds prior to the integration of the sensor package (riskmitigation flights). The risk mitigation flights will provide the opportunity to refine the airframe and manufacturing techniques to increase the survivability as well as capture data on the airframe to improve the final instrumentation integration without putting the instrumentation at risk.

\section{Work Plan Task 2: Requirements Development: Data System and Faraday Fiber Optic Sensor}

Natural lightning strokes and bursts can occur within microseconds to milliseconds, and multiple strokes can occur during a flash, with duration sometimes exceeding 1 second. Peak current amplitudes can be up to 200kA and more, with continuing current of only 100's of amps. The goal is to measure lightning electric current waveforms during attachment to the SE-UAV.

Presently, a two-sensor architecture is envisioned for the genesis SE-UAV, where the fiber loops will cross on the top and bottom of the airframe, to allow determination of electric current flow from wing-wing, wing-front, wing-tail, fronttail, as shown in Figure 5.

Preliminary data requirements are listed here:

- 6 Channels minimum (2 Hi-Speed)

- Hi-Speed Sample Rate $\geq 20$ MSPS

- Hi-Speed Bit Resolution $\geq 12$ bits

- Trigger Setting: Either Hi-Speed Channel, $+/-10$ to $50 \mathrm{mV}$

- Event Capture Duration (i.e. lightning flash): up to 1 second

- Auto-Initialization \& Arming upon power-on, and Auto-Storage and Reset after a trigger event.

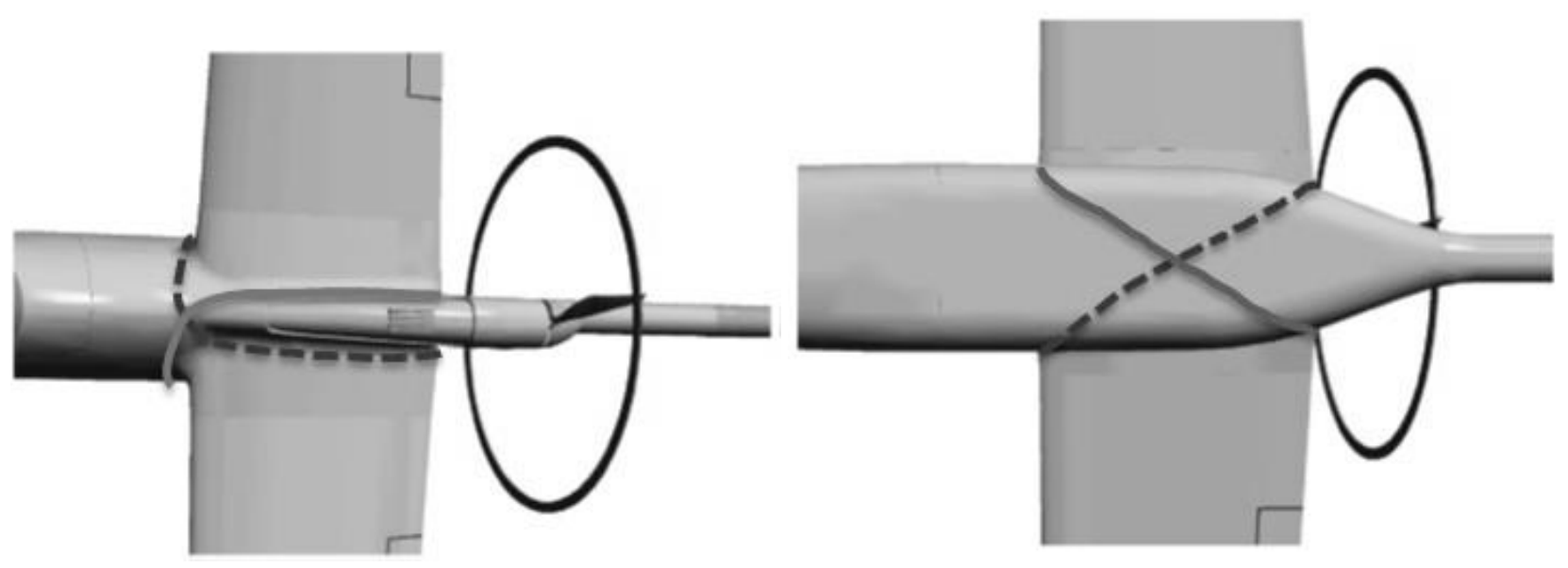

Figure 5. Concept for two fiber-optic loop sensors on top (left) and bottom (right) view of Genesis UAV. 


\section{Work Plan Task 3: Design \& Prototype SE-UAV Data Acquisition System}

AFRC Compact Fiber Optic Sensing System Laboratory, with assistance from LaRC DAQ specialists, will leverage AFRC's state-of-the art UAV instrumentation development experience. A new sFMC architecture allows integration of optical fiber components FPGA digital signal processing, Intel processor, embedded Windows OS, power, and highspeed (Gigabit Ethernet, USB) data interface storage capability.



Figure 6. Representative sFMC 16-Ch. A/D and Power/IO interface stack.

\section{Work Plan Task 4: Design \& Prototype SE-UAV Faraday Sensor System}

A laboratory prototype of the Faraday Fiber-Optic Sensor and a laboratory DAQ system were recently flow on the NASA Falcon HU-25C airplane. The goal was to demonstrate airworthiness and suitable operation in environments where lightning attachment is NOT likely to occur. Confidence in trigger levels and in-flight functionality was obtained. A photo of the HU25C installation is shown in Fig. 7.
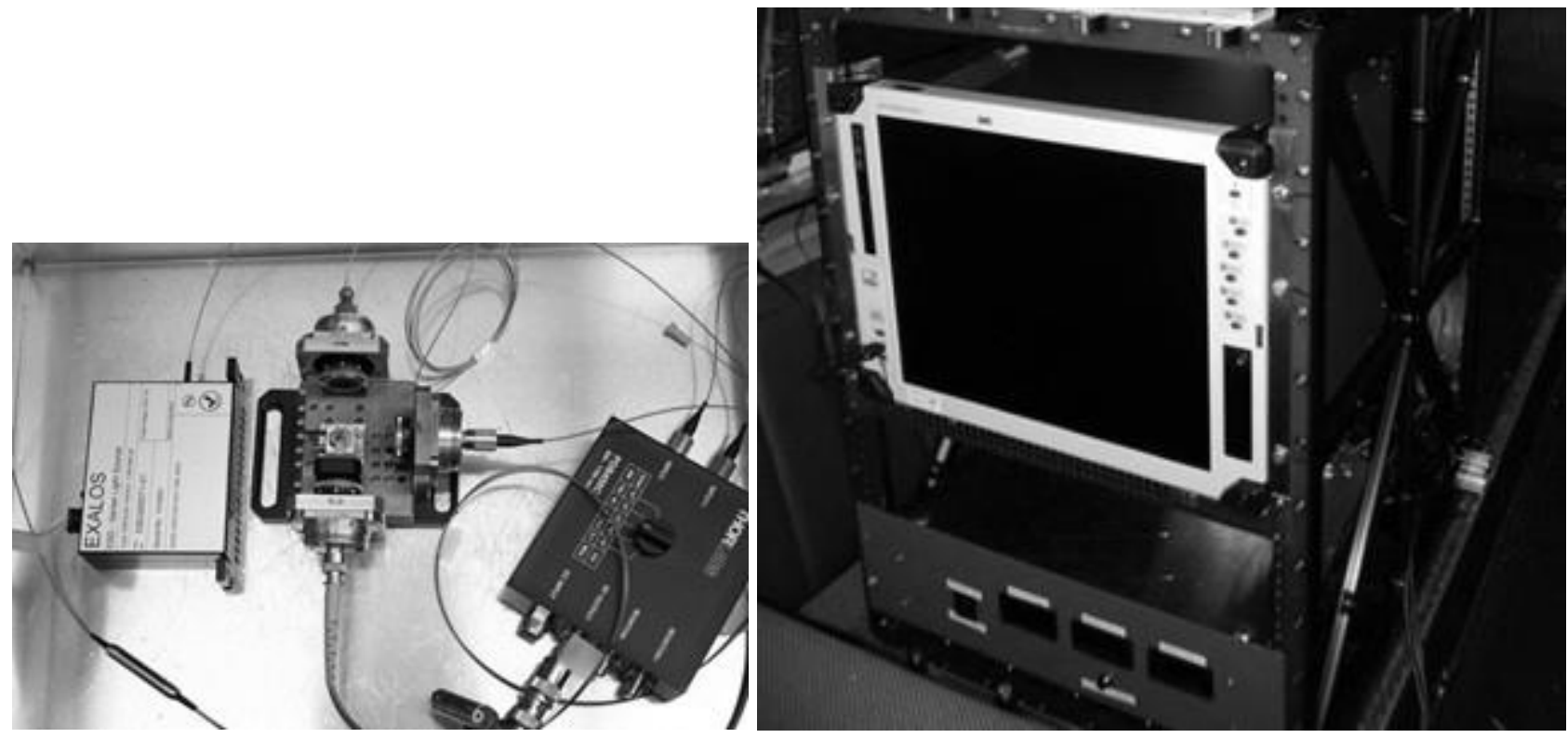

Figure 7. Top: Fiber Optic Faraday Sensor components. Bottom: Laboratory DAQ system and fiber optic Faraday electronics implemented on NASA HU-25C. 
The next step will be to re-package the Fiber Optic system to meet MVP requirements for a UAV, and to ruggedize and harden the system to survive SE-UAV Lightning Attachment. NASA AFRC Compact Fiber Optic Sensing System Laboratory has extensive experience integrating optical fiber amplifiers, detectors, lasers and controllers into the sFMC architecture. (Representative sFMC fiber amplifier board shown in Figure 8.)



Figure 8. Representative sFMC 4-Fiber Amplifiers.

\section{Work Plan Task 5: SE-UAV Payload Fixture Build/Ship to LaRC}

When the final Genesis airframe configuration is determined, a full-scale test fixture of the SE-UAV Payload Experiment Bay will be fabricated and delivered to NASA LaRC to provide a laboratory platform for development and integration of the Faraday sensor and data acquisition system.

\section{Work Plan Task 6: Shielded SE-UAV SN-001 Flight Vehicle Build}

The SE-UAV will be designed and fabricated to accommodate these expected instrumentation requirements:

- $\quad$ Volume $=1 \mathrm{ft}^{\wedge} 3$

- Weight $\leq 20$ Lbs

- $\quad$ Power $\leq 60$ Watts

The SE-UAV may be a scaled version of the Genesis UAV (i.e. 8-10 ft wing span) or be a redesigned mission-specific UAV. The final design will be dictated by the final volume, weight, and power required by the scientific instrumentation.

A flight-ready SE-UAV will be provided to LaRC for integration of the Faraday sensor and data acquisition system and testing as soon as the final UAS design has been completed. This flight vehicle (SE-UAV SN001) will also be used for checkout flights and EMI testing and will have removable wings for transportation between the centers.

Although both UAVs will be designed to meet all flight requirements as needed to complete the scientific mission, SEUAV SN002 will be hardened for the extreme flights through severe weather. The expected mission flight time is 1 hour and in the event of a catastrophic failure after a direct lightning attachment, the UAV will have the capability of deploying a parachute for avionics / instrumentation recovery. 
The final flight vehicle (SE-UAV SN002) will be fabricated in Phase 2 and will be solid without removable parts. The SN002 UAV will have the instrumentation system integrated at KSC for the operational fights currently planned for Phase 2.

\section{Work Plan Task 7: Phase 1 6-Month Status Report}

A 6-Month Status Report will document progress on the Genesis Risk-Mitigation Test Vehicle build, range scheduling \& flight plans, and progress on the prototype SE-UAV DAQ and Faraday Fiber sFMC architecture implementation. ARMD Program management will obtain a clear understanding of progress and likelihood of Phase 2 success.

\section{Work Plan Task 8: Test-Fly Shielded Genesis UAV into Thunderstorms}

The Risk-Mitigation UAV will be flown into KSC-area thunderstorms to demonstrate survivability in the severe environment of an electrified cell. The UAV will be flown through a test sequence decided by the team and recovered (via nominal landing or crash recovery) for analysis. In the event of a direct attachment, the UAV will be retained for post-strike damage assessment and not flown again.

\section{Work Plan Task 9: Build/Integrate \& Test Flight SE-UAV DAQ and Faraday Sensor Systems}

Once the final architecture is determined for the DAQ and optical-fiber Faraday sensor system, a flight prototype will be built and integrated into the Payload Bay Experiment Fixture or SE-UAV SN001 (see Tasks 5 \& 6). A laboratory integration/demonstration test will be performed using standard lightning waveform generating equipment at the NASA LaRC E3-HIRF laboratory.

\section{Work Plan Task 10: Phase 1 Progress Report \& Phase 2 Justification}

Phase 1 Report will include a plan to communicate results at a conference (i.e. SPIE DSS or AIAA), and a NASA Technical Report will include design information for the flight vehicle, DAQ and Faraday system, and how they were hardened for survivability during lightning attachment. The Progress report will also suggest a path for transition into a follow-on NASA (Phase 2) project for lightning, volcanic ash and other aviation hazards.

\section{Work Plan Task 11: KSC Range Analysis, Scheduling \& Support}

Range support will be required for flights of a Risk-Mitigation UAV (newly shielded, likely smaller than SE-UAV SN001 and SN002). But no range analysis or approval will be required to fly within the previously agreed to flight areas and altitudes.

The SE-UAV's will require analysis and approval from the CCAFS 45th Range Safety Analysis group, which can begin when the final design parameters are determined. As part of the project planning for phase 1 it was determined that any Range Safety analysis of the SE-UAV will occur in Phase 2 to coincide with the SN002 build. 




Figure 9. Flowchart describing task work-flow. (Vertical stripes indicate KSC, Horizontal stripes indicate AFRC, and gray shading indicates LaRC involvement.)

\section{Deliverables}

The Phase 1 effort will result in a flight-proven Genesis UAV, hardened for operation in Florida thunderstorms, and ready for installation of the data acquisition system and Faraday fiber optic sensor system. The final Phase 1 report will be a NASA Technical Report, and will include design information for the flight vehicle, DAQ and Faraday system, and how they were hardened for survivability during lightning attachment.

1. 3 Months: Genesis Payload Fixture

2. 6 Months: Phase 1 Status Report

3. 8 Months: Shielded SE-UAV

4. 10 Months: Flight DAQ and Faraday Fiber-Optic Sensor System integrated into SE-UAV SN-001

5. 12 Months: Phase 1 Final Report \& Phase 2 Justification. 


\section{NASA'S AERONAUTICS MISSION AND BEYOND}

In 2014, NASA ARMD rolled out a new strategic vision that focuses on six strategic thrust areas [6]:

1. Assured Autonomy for Aviation Transformation

2. Innovation in Commercial Supersonic Aircraft

3. Ultra-efficient Commercial Vehicles

NASA ARMD Strategic Thrusts

4. Transition to Low-Carbon Propulsion

5. Real-time System Safety Assurance

6. Safe, Efficient Growth in Global Operations

ARMD reorganized four mission programs to define the compelling technical challenges facing the aviation industry, act upon those challenges, and modify them as required by NASA's stakeholders and customers. The programs are:

1. Airspace Operations and Safety Program (AOSP)

2. Advanced Air Vehicles Program (AAVP)

NASA ARMD Programs

3. Integrated Aviation Systems Program (IASP)

4. Transformative Aeronautics Concepts Program (TACP)

TACP is subdivided into three Projects: Convergent Aeronautics Solutions (CAS), Transformational Tools \& Technologies (TTT), and Leading Edge Aero Research for NASA (LEARN). CAS focuses upon short-duration activities to establish early-stage concepts and technology feasibility. Internal teams propose ideas, conduct feasibility studies, perform experiments and try out new ideas in a 1-year cycle. ARMD reviews and selects the most promising capabilities for further development by other NASA aeronautics programs or by direct transfer to the aviation community.

The ARMD reorganization terminated aviation safety-focused research activity related to aircraft sensing of atmospheric environmental hazards, and aircraft sensing for health management. This paper can be viewed as an effort to establish the importance of in-situ and remote sensing of flight environment in the context of autonomous flight technologies. The authors of this paper proposed to a 2014 CAS Seedling call with the SE-UAV concept, and continue to pursue the concept as a way to expand vehicle capability and airspace access to enable development of emerging sensors, structures and flight controls to operate in extreme flight conditions (storms, volcanic plumes, icing, forest fires, radiation, etc.). In order to establish trust in autonomous operations, UAVs need to compensate for the absence of a pilot with increased resiliency to upset conditions, necessitating improved situational awareness of the environment. (i.e. Strategic Thrust \#1: Assured Autonomy for Aviation Transformation)

A NASA SE-UAV Test fleet will function as a test platform for sensor-perception technologies that will enable UAS performance that surpasses human-piloted capability in hazardous flight conditions. UAS for research, science, and search \& rescue will become a trusted part of the NAS, and be fully dependable in severe environmental conditions. Routine, on-time UAV cargo operations will be possible in weather conditions that are too uncomfortable for passenger airplanes. This work will lead to general aviation and corporate jets, augmented by on-board autonomy, that will operate safely and reliably in any NextGen 4D ATM environment and beyond. (i.e. Strategic Thrust \#6: Safe, Efficient Growth in Global Operations) 


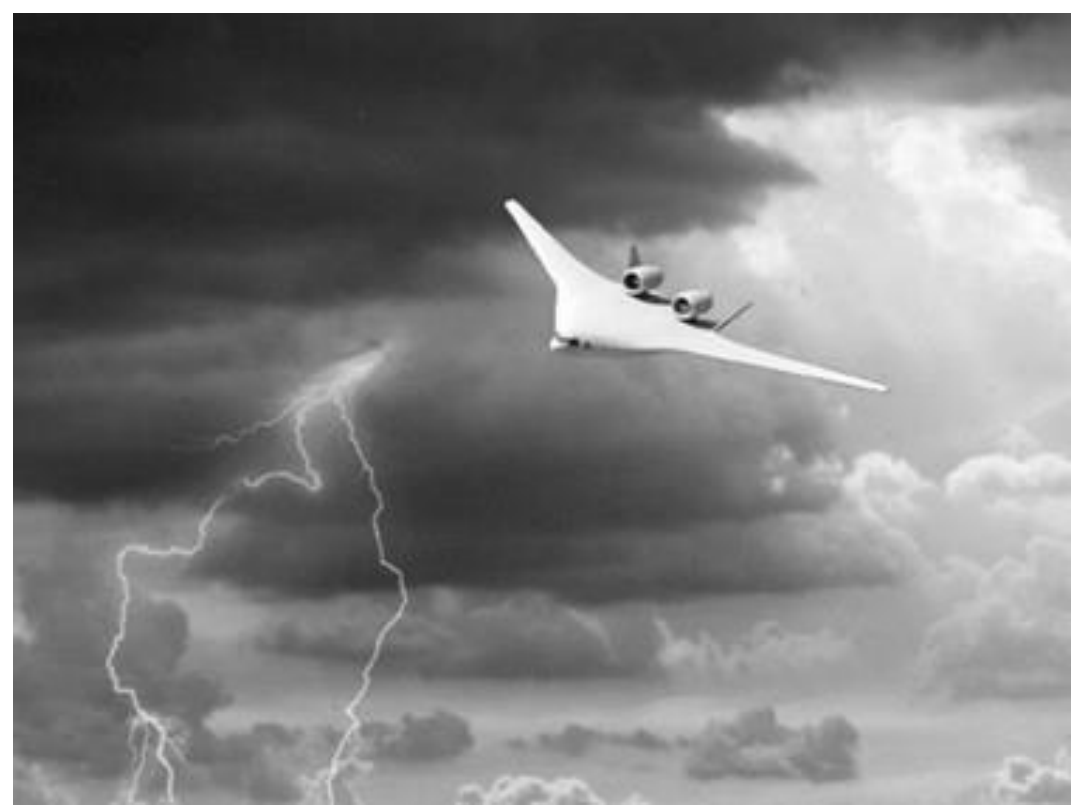

Figure 2. Graphic artist concept of a Cargo UAV performing routine operations in a thunderstorm- on schedule regardless of weather conditions.

\section{NASA ARMD "Big Questions"}

In order to compare and prioritize proposed research projects that address the ARMD Strategic Thrusts, examples of "Big" research questions (or objectives) have been formulated. These Big Questions are not intended to be exclusive, but rather provide examples of how proposed research efforts can directly impact the Strategic Thrusts. Timely examples of 10 Big questions can be found at [7]. These are provided below, along with how SE-UAV operations address Big Questions \#9 and \#10.

1. How can energy consumption for a given phase of flight or speed regime be minimized?

2. How can any class of Unmanned Aircraft System (UAS) be permitted to operate in any part of the National Airspace System (NAS) where there is a legitimate need without constraints beyond those already mandated for manned aircraft?

3. How can General Aviation be made simultaneously safe, convenient, and affordable for a greater portion of the population?

4. How can the three key environmental barriers to public acceptance of commercial supersonic flight, sonic boom, emissions, and community noise, be overcome?

5. How could aircraft be custom build or modified "on-demand" for specific market and/or mission requirements at low cost?

6. How can automated and autonomous airborne and ground flight management systems be designed and implemented in a way that ensures the safety of operations and the productivity of the system, while maintaining operator proficiency at a level necessary to deal with unforeseen circumstances?

7. What is the optimum combination of low cost, environmentally benign, light weight, efficient, compact electrical power generation for either propulsion or onboard systems?

8. How can the NAS be significantly improved to be better optimized for efficiency, safety, and convenience?

9. How can trust in autonomous systems be established? 
$>$ Inexpensive and resilient, SE-UAVs (and their derivatives) will enable operations and science research in severe atmospheric environments that are normally avoided by crewed aircraft. SE-UAVs will provide a platform for in-situ and remote sensing, as well as verifying that resilient autonomous systems are behaving as intended. These new missions logically lead to disaster assessment and search $\&$ rescue missions. As SE-UAV operations become more common and valued by the public, regulatory accommodation of UAVs will increase. In-situ SE-UAV research data will one day make routine, on-time UAV cargo operations possible in weather conditions that are too uncomfortable for passenger airplanes. UAVs will become a trusted part of the NAS, and will be fully dependable in severe environmental conditions. This Big Question is associated with the Real-time system-wide safety assurance and Assured autonomy for aviation transformation Strategic Thrusts.

10. What new missions with the potential to transform the air transportation system could be enabled through the application of new and emerging technologies?

> New lightning environment data resulting from SE-UAV flights into thunderstorms will be directly usable for certification of UAV's and passenger airplanes. An improved understanding of severe weather processes is required to expand capacity of the global airspace system and save time and fuel. Powerful remote sensing tools are in-service and under-development (i.e NEXRAD, NLDN, GOES-R, LMAs, WXR, etc.), but full utility of their data is incomplete without in-situ measurement of actual conditions in severe environments normally avoided by aviators. NASA SE-UAVs will enable testing new radar and lidar systems to detect turbulence, windshear, wake-vortices and hail; machine vision for fusion of visible/IR/radar imagery and neurally-inspired hazard recognition; and measuring XRays, Gamma-Rays, and other electromagnetic emissions that have been show to correlate directly to turbulence and hydrometeor formation inside storms. NASA SE-UAVs will provide a capability for testing innovative structures, health management and flight control systems for routine operation in extreme environments. As a new tool for understanding severe weather processes, and as a platform for testing new sensors technology, structures, flight control and health management, SE-UAVs have the potential to transform the air transportation system. This Big Question is associated with the Safe, efficient growth in global operations and Assured autonomy for aviation transformation Strategic Thrusts.

\section{Beyond NASA ARMD}

While the concept is compelling from a NASA Aeronautics mission perspective, SE-UAV operations could demonstrate sensor technologies and extend meteorological science missions of the Storm Penetrating Aircraft (an A-10 Thunderbolt, funded by the National Science Foundation, and operated by the South Dakota School of Mines in Norman, Oklahoma).

The present SE-UAV concept is planned to incorporate a tiny electric field sensor developed under NASA Small Business Innovative Research (SBIR) project for a previous UAV flight campaign. We believe that DOD, DARPA and the academic lightning research community may have strong interest in follow-on, concurrent UAV measurements of electric field, current and X-Rays associated with lightning.

The FAA and SAE-AE2 Lightning Committee continue to refine and document the lightning environment for airplanes. New statistical data for lightning waveforms- peak amplitude, energy profile, and frequency content that will be enabled by SE-UAV's will greatly extend this understanding, and improve confidence in the certification environment for air vehicles. 


\section{IN CONCLUSION}

A SE-UAV concept that can perform in-situ measurements in hazardous atmospheric conditions has been described. State-of-the-art capability is leveraged from three NASA centers to develop a proof-of-concept vehicle and measurement system that can survive lightning attachment during flight operations into thunderstorms. The SE-UAV will provide inflight lightning electric-current return stroke and recoil leader data, and serve as a platform for development of emerging sensors and new missions into hazardous environments. An overview of the NASA Aeronautics Strategic Vision, Programs was provided, along with how a SE-UAV is envisioned to impact them. NASA's Aeronautics and Science Missions have expressed interest in a capability to perform in-situ volcanic plume measurements and long-endurance UAV operations in various weather conditions. The SE-UAV concept is presented in the SPIE DSS venue to explore teaming, collaboration and advocacy opportunities outside NASA.

\section{REFERENCES}

[1] F. Pitts, B. Fisher, V. Mazur, and R. Perala, "Aircraft Jolts from Lightning Bolts," IEEE Spectrum, July 1988.SAE Lightning Environment Doc

[2] ARP-5412 “Aircraft Lightning Environment and Related Test Waveforms," Rev. B, Jan 2012.

[3] V. A. Rakov, M. A. Uman, [Lightning: Physics and Effects], Cambridge University Press, The Edinburgh Building, Cambridge CB2 2RU, UK, (2005)

[4] "Genesis Aircraft Review" Airworthiness and Flight Safety Review Board (AFSRB) Presentation, Flight Systems Engineering Training Program- Unmanned Aerial Systems Laboratory, Kennedy Space Center, August 15, 2013.

[5] Nguyen, T. X., Ely, J. J., Szatkowski, G. N., "A Fiber-Optic Current Sensor for Lightning Measurement Applications", Proc. SPIE

[6] K. Rugg (Editor), T. Springer (NASA Official), "Aeronautics Research Mission Directorate Programs", $<$ http://www.aeronautics.nasa.gov/programs.htm>, 25 March 2015

[7] D. Bazar (Editor), M. Dudley (NASA Official, "ARMD Strategic Thrusts", < http://www.nari.arc.nasa.gov/thrusts>, 25-March-2015 